\title{
An estimate of the short-term local climatic changes by measuring the air temperature, air pressure and humidity
}

\author{
Anton Stoilov and Borislav Yurukov \\ South-West University "Neofit Rilski" \\ Bulgaria
}

\begin{abstract}
This paper presents an assessment of local climate changes in Southwestern part of Bulgaria, specially region of Blagoevgrad. In this study was conducted research and measurement of weather parameters such as air temperature, humidity and air pressure. The analysis is carried out for a month of July in the period of last five years (20112015). Hardware is unchanged and the main basic sensors are the same throughout the study period. Weather station used for this scientific research was WS-2355. In practice, the changes are analyzed and given an assessment of climate change in the past five years. An attempt to found new trends and identify dependencies for predicting the next climatic parameters. Relatively few data on such changes still can not give an unambiguous assessment of climate change, but the collection of local data is a step towards solving this scientific problem.
\end{abstract}

Keywords - measuring, air temperature, air pressure and humidity, climatic changes;

\section{Introduction}

Advancing climatic changes lead to changes in the conditions of real life on the Earth. Studies have present that they are the result of increasing average surface temperatures on Earth as a result of different factors. In 1997, the governments of industrialized countries adopted the Kyoto Protocol on the issues of climate change. Protocol affect virtually all major sectors of the economy and is considered the most extensive agreement on environmental issues and sustainable development, signed so far. Bulgaria ratified the Kyoto Protocol on 17 July 2002, which joins the efforts of the international community to combat climate change [1]. Completed and detailed description of climate change and the impact of these changes on humans and nature, is given in the prepared evaluation reports of the Intergovernmental Panel on Climate Change (IPCC). The third IPCC report from 2001 shows that the global average surface temperature has risen by about $0,6^{\circ} \mathrm{C}$ per century and that "... most of the observed warming over the last 50 years is attributable to human activities" [2]. According to the Fourth Assessment Report of the IPCC, released in 2007, the average global temperature of the Earth is now $15^{\circ} \mathrm{C}$ and the warming trend is to continue in the XXI century with much higher rates; the level of the seas and oceans rise, most glaciers in temperate regions consistently reduced their volumes [3]. On the territory of Bulgaria climate changes follow global trends-minimum temperatures have risen by an average of $0,6^{\circ} \mathrm{C}$ over the past 100 years, there was a reduction in the amount of rainfall is expected in the 20 years of this century, they diminish with about $10 \%$, which will lead to a significant reduction in the overall water reserves. The climate is a multi-mode time statistical characteristic of an area depending on its geographical position. Studied by means of long-term measurements of meteorological elements and determine their average condition. Such measurements have been made for more than 300 years, not only on land but also in the higher parts of the atmosphere and ocean. The collected data is used to determine the status of the multi-annual average standby time.

\section{Method}

South-West University "Neofit Rilski" (SWU) at latest several years making significant efforts in the district of collecting meteorological data. As a major scientific and educational center, SWU adopted as its mission to provide reliable experimental data to estimated global and local climatic changes and maintaining a network of weather stations. As a result of these efforts was developed a weather network in Southwestern Bulgaria in 7 different cities (Kyustendil, Dupnitsa, Blagoevgrad, Sandanski, Petrich, Gotse Delchev and Razlog) were installed automatic weather stations (AWS) of the type WS-2355 company LaCrosse. The stations were configured to measure the main parameters of the environment and to send the measurement data to the our server of the South-West University. So allow for real-time tracking of climate processes and after collecting enough data - conducting analysis of climate change and assessing future trends and forecasts. This article try to presents one such assessment of local climate change for past five years - from 2011 to 2015 . The huge array of experimental data which are storage, we have selected data for month July for each of the aforementioned period of time. Choosing of this month was made because if we want to observed effects from global warming, then it will be best expressed and shown in the hottest month of the year for latitudes of Bulgaria, namely July. On the other hand compensatory mechanisms of nature can mitigate the potential effects of warming in average values of climatic parameters, but during the hottest month of the year, if this phenomena existed, such effect he would be the most recognizable. In practice, if you look clearly and categorically proof of global warming and its effects, then for the region of Bulgaria are experimental data in July, followed chronologically. Environmental parameters that are really dependent on local climatic changes are air temperature first, atmospheric pressure - as an indicator of more intense and turbulent processes and humidity - as a generalized indicator of the amount of moisture. It is reasonable to ask why not read the quantity of rain as a primary parameter of the effect of global warming. Meet is that the hottest months rainfall default occasional 
Proc. of the Third Intl. Conf. on Advances in Bio-Informatics and Environmental Engineering - ICABEE 2015

Copyright @ Institute of Research Engineers and Doctors, USA .All rights reserved.

ISBN: 978-1-63248-078-1 doi: 10.15224/ 978-1-63248-078-1-06

disturbances are extremely rare events. This makes this climate parameter is not sensitive enough and suitable for expression of local climate change. Conversely, the amount of water in the air, which is extremely sensitive climatic parameter would show a real change in local weather map. Therefore this publication suggests that assessment of local climate change is the most appropriate to measurements made in the hottest month of the year (if you desired to study effects of global warming) and to make precise measurements of air temperature, atmospheric pressure and humidity. Of course, a comprehensive study was seized multiple measuring points for better visibility of the effects of climate changes. This study, however, aims to show local effects, if any, and possibly in subsequent publications to summarize local variations. So here are presented only data from the automatic weather station in city of Blagoevgrad, where is the headquarters of our university.

\section{A. Hardware}

A weather station is a facility, either on land, with instruments and equipment for observing atmospheric conditions to provide information for weather forecasts and to study the weather and climate. The measurements taken include temperature, barometric pressure, humidity and etc. The base station LaCrosse Ws-2355 measures the indoor environment of its surrounding area and receives weather data from the following three outdoor sensors: ThermoHygro Sensor, Wind Sensor and Rain Sensor. Specifications of weather station WS-2355 is present on Table.1.

\section{TABLE I. SPECIFICATIONS OF WEATHER STATION WS-2355}

\begin{tabular}{|l|r|}
\hline \multicolumn{1}{|c|}{ Parameter } & \multicolumn{1}{|c|}{ Value } \\
\hline Temperature Range & $29,9^{\circ} \mathrm{C}$ to $+69,9^{\circ} \mathrm{C}$ \\
\hline Temperature resolution & $0,1^{\circ} \mathrm{C}$ \\
\hline Measuring Range Rel. Humidity & 0 to $999,9 \mathrm{~mm}$ \\
\hline Rain Volume & $0,1 \mathrm{~mm}$ \\
\hline Rain resolution & 0 to $180 \mathrm{~km} / \mathrm{h}$ or 0 to $50 \mathrm{~m} / \mathrm{s}$ \\
\hline Wind speed & $0,1 \mathrm{~m} / \mathrm{s}$ \\
\hline Wind speed resolution & $8 \mathrm{~seconds}$ \\
\hline $\begin{array}{l}\text { Measuring interval } \\
\text { thermo-hygro sensor }\end{array}$ & \\
\hline
\end{tabular}
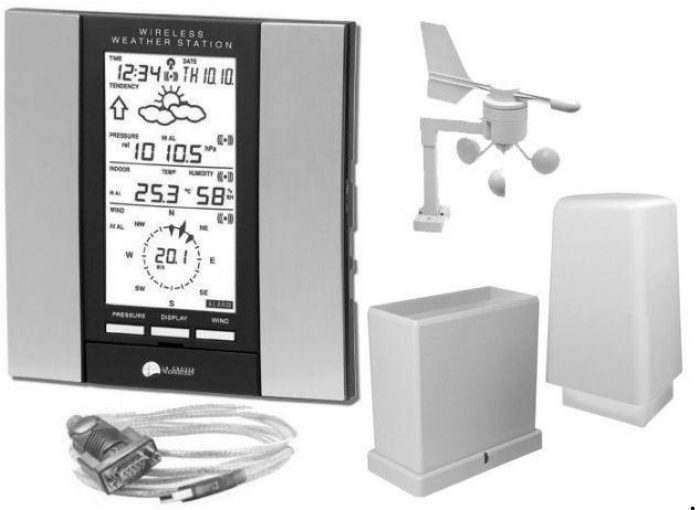

Figure 1. Weather station WS-2355
The received data is continuously updated to bring you the latest weather information on the base station's LCD. The thermo-hygro sensor is the main data communication unit since both the wind and rain sensors are connected to the thermo-hygro sensor for operating power and rely on it to communicate to the base station. Weather data sent from the thermo-hygro sensor can be done by wireless $433 \mathrm{MHz}$ transmission. The thermo-hygro sensor measures the outdoor temperature and relative humidity. It also collects the readings from the rain and wind sensors before transmitting the data to the base station by wireless $433 \mathrm{MHz}$. The wind sensor measures wind speed and wind direction and sends the data to thermo-hygro sensor which in turn transmits the data to the base station. Operating power is taken from the thermo-hygro sensor using a $10 \mathrm{~m}$ cable connection. The rain sensor measures the rainfall and sends the data to thermo-hygro sensor which in turn transmits the data to the base station. Operating power is taken from the thermo-hygro sensor by a $10 \mathrm{~m}$ cable connection [4].

\section{B. Mounting of weather station}

The installation of the sensors is one of the most important conditions for accurate measurements of meteorological scientific purposes. In this study the sensors were installed at two meters height, above the ground, on graced area. When installing the sensors have met all the requirements and dependencies (fig.2.). In order to better transmit data from the transmitter to the receiver with direct visibility between these two modules - receiver and transmitter.

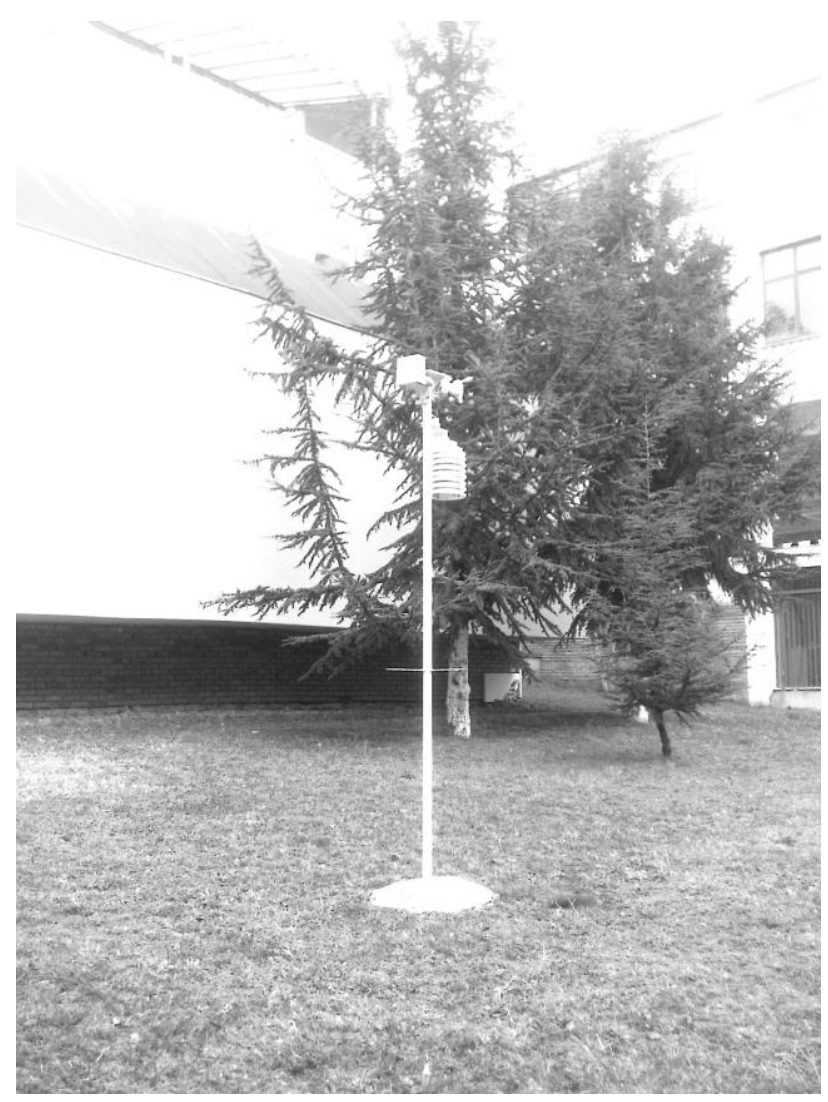

Figure 2. Picture from basic sensors mounting 
The transmitter is powered by batteries, which provides both energy independence and protection from lightning. Also the main carrier mast (tube) is grounded again to protect against lightning and thunder. Supporting structure is mounted on a concrete pedestal. Compliance with these requirements ensure and guarantied the representativeness of the data. Weather station is located in the courtyard of South-West University (Fig.1). Sensors are located 2 meters above the grass. Thermo-Hygro Sensor is located in the Stevenson screen. Receiver module is located 40 meters line of sight from the transmitter in a specialized laboratory. Coordinates of measurement unit are $42^{\circ} 01^{\prime \prime} \mathrm{N}$ and $23^{\circ} 02^{\prime \prime} \mathrm{E}$. This weather station consists of a base station and three sensors and software for use to upload all weather data from the base station to the Internet. The base station measures the indoor and receives the outdoor weather information from 3 outdoor sensors (thermo-hygro, wind and rain sensors).

\section{Results}

The results of measurements with automatic meteorological station are presented below. They cover monthly reports five years ago for July. The results are copyrighted and are the fruit of the efforts of both authors for construction, maintenance and renewal of the system of measurement. Experiment was conducted under identical conditions of hardware and software, transmission speed and manner are the same. All this allows us to compare the results obtained and draw conclusions. This type of experiments require perseverance and tenacity, so rare in the scientific literature.

\section{A. Air Temperature}

Once you have made accurate measurements of air temperature by automatic measuring station Blagoevgrad, Bulgaria, the results are presented in graphical form in Figure 3. The chart shows five different lines corresponding to five consecutive years in which measurements were made for July. Measurements include minutely temperature values, then averaging is performed on all entries of the day - the points on the graph. Each day is represented by a value, is then passed regression curve to smooth the graph. Used polynomial of 2 nd degree. Thus is presented correctly monthly trend change in temperature in Blagoevgrad by average daily temperatures. Along with the graphical presentation was conducted and numerical analysis of data. Thus it is made, and analysis of variance of the deviations and errors. Given the limited volume of this publication distributional analysis was not present, but can be provided if it is necessary. As can be found from the graph have exactly two basic models of the behavior of the air temperature.

The first model include 2011, 2012 and 2015. In this model have air temperatures around $27^{\circ}$ Celsius, with deviations of 2 degrees Celsius than it gets. Unimpressive relatively similar course of air temperature during the month for different years in the same range. This suggests that this is one of the main trends in the move The temperature for July. These temperatures are not extremely high and far from temperature records, which once again confirms the representativeness of measurements. The homogeneity of the points showed a clear trend and pattern of temperature profile.

The second model include 2013 and 2014 years. There are observed at lower levels around $21-22^{0}$ Celsius. The difference with the previous model is significant and obvious. The temperatures are lower and the points are homogeneous ie we have again a clear trend in the temperature profile. This requires the conclusion that this is the second major trend in the temperature move. Since two years are adjacent obviously we have resistance to this trend.

The data presented in practice show that local climatic changes are not significant, since alternate sequences of two years in alternating warmer to colder periods. This finding was confirmed in a longer period, which goes beyond the scope of this article. The fact that the periods are not long two years shows that we have a balance globally.

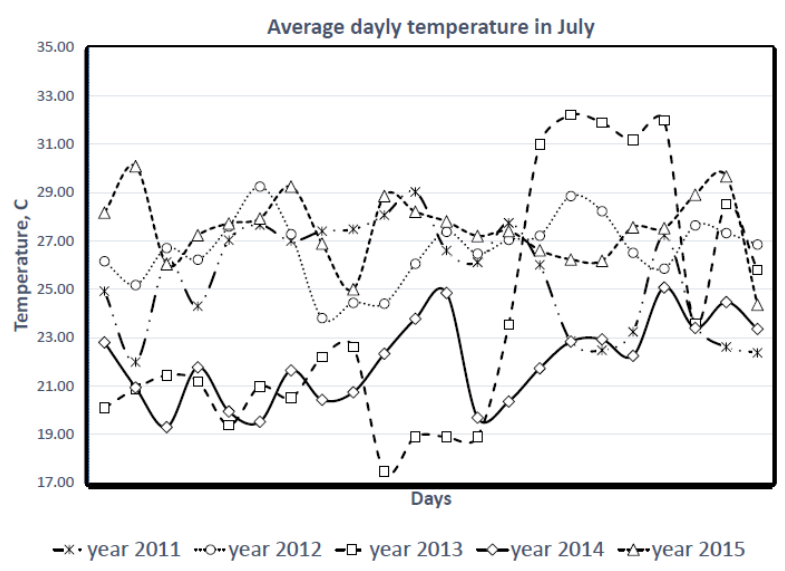

Figure 3. Graph of temperature in July (2011-2015)

\section{B. Air pressure}

Such as temperature measurements were conducted also and air pressure measurements (fig.4). Here more clearly outline the two main climate models. Differences in climate are now reflected as different average daily pressures. As mentioned above pressure is a dynamic parameter and the integration gives a more accurate picture of the local features. This fi explanation for even better shape of the two climate models. The first model again covered 2011, 2012 and 2015 years, the value of the average pressure is about $1010 \mathrm{hPa}$. Values are stable throughout the month, which determines the lack of turbulent changes in external air card. This finding further confirms the selection of the July examination local climate change. Second model again covers 2013 and 2014 years. The atmospheric pressure in this period is substantially lower than the other three and is approximately $965 \mathrm{hPa}$. Unlike $50 \mathrm{hPa}$ at average value is significant and shows various weather conditions. Again suggests that alternate two models - high pressure and low pressure. The lack of a direct relationship between air temperature and atmospheric pressure, but recognizing identical trends proves the representativeness of the data. The two climate models that alternate every two years is increasingly emerging. 
Pressure as a physical parameter is relatively independent indicator of human activity, so that the conclusions are anthropogenic independent. The two climate models clearly shown in the graph in Figure 4. give a new approach to define persistent relationships and predictions based only on measurements of atmospheric pressure.

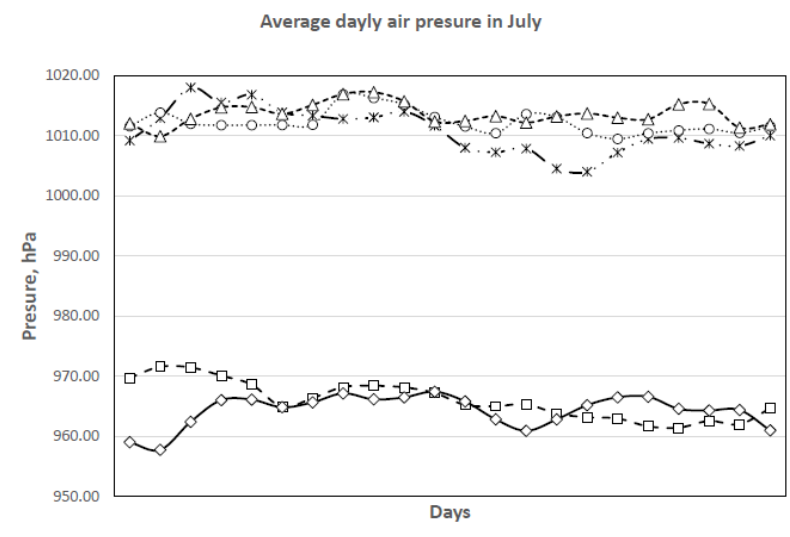

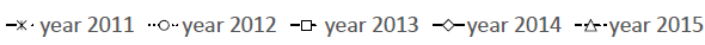

Figure 4. Graph of air presure in July (2011-2015)

\section{Humidity}

Humidity is an important environmental parameter that takes into account the cumulative impact of all types of rainfall and the irrigation and soil moisture. All these parameters are very hard to be measured separately and appreciate. Presented in Figure 5 graphs show averages of humidity for each day of the month of July for five years. Again both weather model is clearly visible - the first enclosing 2011, 2012 and 2015 and second 2013 and 2014. In the first model the humidity is significantly higher than the second with about $20 \%$ on average, which is significant. Given the choice of the hottest month of the year in contrast to $20 \%$ of impact significant environmental parameters and in particular human activity. In literature, periodic measurements of humidity are not very popular and common.

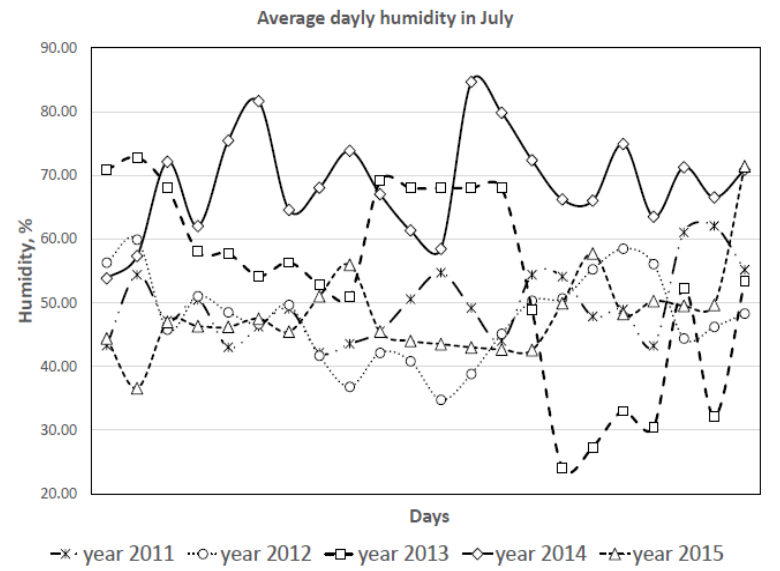

Figure 5. Graph of humidity in July (2011-2015)

As can be seen from our results, however, this climatic parameter is also shown climate change. Humidity gives us an integrated assessment and this makes it very important in determining to weather changes.

\section{Conclusions}

Current article is a new approach for assessing local climate change. Used approach in measuring a time interval for tracking changes in the air temperature, atmospheric pressure and humidity. It has been shown that these parameters can fully identify and separate recognized weather patterns and trends. As a result of the specific research imposed following conclusions:

1. The data obtained from the measurements do not confirm the existence of global weather warming in the short time term for Southwestern Bulgaria.

2. Alternating periods of higher air temperatures to those with lower air temperatures regularly

3. Conducting measurements with local impact of the basic meteorological parameters can be used to draw conclusions that can be summarized globally.

\section{Acknowledgment}

This research was supported by project:

Bioinformatics Research: Protein Folding, Docking and Prediction of Biological Activity NSF - 02/16 funded by National Research Fund of Bulgaria and South-West University "Neofit Rilski"

\section{References}

[1] http://www.moew.government.bg/

[2] IPCC's Third Assessment Report AR3, 2001.

[3] IPCC's Fourth Assessment Report AR4, 2001.

[4] Operation Manual Professional Remote Weather Station WS-2355

[5] Allen, R. G., Brockway, C. E., and Wright, J. L. 1983. Weather station siting and consumptive use estimates. J. Water Resour. Ping, and Mgmt. Div., ASCE 109 (2): 134-146.

[6] Fuchs, M., and C. B. Tanner, 1965: Radiation shields for air temperature thermometers. J. Appl. Meteor., 4, 544-547.

[7] Nachtigal, C. L., 1990: Instrumentation and Control: Fundamentals and Applications. Wiley-Interscience, $890 \mathrm{pp}$.

[8] Tanner, B. D., 1990: Automated weather stations. Remote Sens. Rev., 5, 73-98.

[9] Hubbard, K. G., and X. Lin, 2002: Realtime data filtering models for air temperature measurements. Geophys. Res. Lett., 29.

About Author (s):

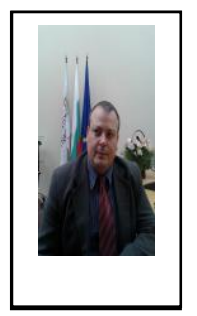

Assist. Prof. Anton Nanev Stoilov, PhD South-West University "Neofit Rilski”, Blagoevgrad, Bulgaria, was born on 10 Nov 1977 in Stara Zagora, Bulgaria. Scientific interest are in field of Measurements of physical, chemical and biological values. 